\title{
Factors Associated with High Mortality of Gastric Adenocarcinoma in Thailand Versus Japan
}

\author{
Rapat Pittayanon ${ }^{1}$, Noriya Uedo ${ }^{2}$, Thapanee Praipisut ${ }^{1}$, Yusuke Tounai ${ }^{2}$, Rungsun \\ Rerknimitr $^{1}$, Pinit Kullavanijaya ${ }^{1}$
}

${ }^{1}$ Division of Gastroenterology, Department of Medicine, Chulalongkorn University and King Chulalongkorn Memorial Hospital The Thai Red Cross, Bangkok, Thailand. ${ }^{2}$ Department of Gastrointestinal Oncology, Osaka Medical Center for Cancer and Cardiovascular Diseases, Osaka, Japan.

\begin{abstract}
Background and aim: Thailand and Japan have high prevalence of Helicobacter pylori infection but prevalence of gastric cancer and mortality rate are difference. Most international comparative studies are epidemiologic studies and the difference in actual clinicopathological data has not been clarified. This study aimed to investigate the difference in clinicopathological characteristics of gastric adenocarcinoma between Thailand and Japan.

Methods: This retrospective study was conducted in two high-volume hospitals for gastric cancer in Thailand and Japan. Baseline characteristics, clinical manifestations, tumor characteristics, treatments and mortality rate of gastric cancer patients in the two institutions were examined. Results: A total of 403 Thai and 2,318 Japanese patients were retrieved from the electronic database. Finally, 332 Thai and 414 Japanese patients (randomly sampled) with gastric adenocarcinoma were eligible for analysis. Thai gastric adenocarcinoma patients were significantly younger than the Japanese $(59 \pm 13$ vs. $68 \pm 10$ years old, $\mathrm{p}<0.001)$ and the majority of Thai patients were female $(55 \%$ vs. $25 \%, \mathrm{p}<0.001)$. The rate of smoking $(5.1 \%$ vs. $23 \%, \mathrm{p}<0.001)$ and alcohol drinking $(2.7 \%$ vs. $34 \%, p<0.001)$ were lower in Thai patients when compared with those in Japanese. The mortality rate at the conclusion of this study (July 2016) was significantly higher in Thai patients $(75.3 \%$ vs. $7 \%, p<0.001)$. In multivariate analysis, ethnicity, clinical UICC stage II-IV compared to I, and resection-based treatment were significant prognostic factors after adjusting for age, gender, presence of alarm symptom, lesion extension and histology. Conclusion: Thai patients with gastric adenocarcinoma were different from Japanese patients in all aspects. Advanced stage of disease, country, and un-resected tumor were associated with poor prognosis. An individual strategy to improve survival of gastric adenocarcinoma in a low-prevalence area such as Thailand should be explored.
\end{abstract}

Keywords: Gastric cancer- Thailand- Japan

Asian Pac J Cancer Care, 3 (2), 29-35

\section{Introduction}

Gastric adenocarcinoma remains a health-care problem all over the world due to its high mortality rate $[1,2]$.Thailand has low prevalence of gastric adenocarcinoma; however, the mortality rate is high because patients usually present with advanced stage of disease[3]. In contrast, although there is a high prevalence of gastric adenocarcinoma in Japan, the mortality rate is low because of early diagnosis and prompt curative treatment by endoscopic and surgical resection [4]. In countries where gastric cancer screening is implemented
Submission Date: 02/21/2018 Acceptance Date: 05/05/2018

i.e. Japan and Korea, mortality rate is about half of the incidence rate [5]. In contrast, mortality rate is very close to the incidence rate in other Asian countries, including Thailand [5]. Early gastric cancer detection strategy by endoscopy may contribute to better survival rate in those countries [6-8]. However, most international comparative studies were conducted based on epidemiological data [9] and studies to compare actual clinicopathological data have been lacking.

According to the recommendation from National Cancer Institute (NCI), there was no evidence that gastric cancer screening program by endoscopy would increase

Corresponding Author:

Dr. Noriya Uedo

Department of Gastrointestinal Oncology, Osaka Medical Center for Cancer and Cardiovascular Diseases, Osaka, Japan.

Email: uedou-no@mc.pref.osaka.jp 
survival rate in low prevalence area of gastric cancer. [10] Moreover, screening endoscopy in low incidence countries is not efficient and cost-effective because of the low detection rate of gastric cancer despite large numbers of screening endoscopy [10]. The differences in patient's clinicopathological characteristic between high and low prevalence of gastric cancer region should be further examined to elucidate factors that associate with poor survival rate of patients with gastric cancer. Therefore, this study aimed to evaluate characteristics of patients with gastric adenocarcinoma and factors associated with different outcome in Thailand and Japan to find a clue to improve patients' survival in low prevalence country such as Thailand.

\section{Materials and Methods}

Study design, patient population, and data collection

This is a retrospective cohort study. It was conducted at King Chulalongkorn Memorial Hospital, Thailand and Osaka Medical Center for Cancer and Cardiovascular Disease, Japan. This study was registered and approved by the institutional review board in each institution and ClinicalTrials.gov (NCT03059732).

\section{Participants}

Eligible cases were selected by formal computer searches of all patients seen at out-patient clinic and/or hospitalized for gastric cancer between 2010 and 2014. The exclusion criteria were 1) age $<18$ years, 2) incomplete data, 3) missed diagnosis of gastric adenocarcinoma and 4) died from other causes (not related to gastric adenocarcinoma).

\section{Variables}

Age was defined as the age at diagnosis of gastric adenocarcinoma. Current smoking and alcohol drinking were defined as smoking or drinking at the time of gastric adenocarcinoma diagnosis. Alarm symptoms were noted if the patient had one of the followings; 1) early satiety/abdominal fullness, 2) gastrointestinal bleeding, 3) severe vomiting, 4) anemia, 5) dysphagia, and 6) unexplained weight loss (in 3 months). H. pylori infection was defined as at least one positive test amongst rapid urease test, urea breath test, serum antibody test, and pathology. Tumor characteristics were tumor location in stomach, in longitudinal and circumferential aspect, and its pathological type. Longitudinal locations were 1) upper (fundus), 2) middle (body) and 3) lower (antrum). Circumferential locations were 1) anterior, 2) posterior, 3 ) lesser curvature, and 4) greater curvature. The lesion extension was then categorized into extension of $<2$ and $\geq 2$ of longitudinal or circumferential areas. Pathology was derived from the report in each institute. In Thailand, histology was classified according to WHO classification (11) whereas Japanese pathologists used their own classification (12). Union for International Cancer Control (UICC) staging in this study was from the 7th edition UICC (13) and clinical stage was assessed. Treatments were categorized into endoscopic resection, surgical resection, surgical resection plus chemotherapy (CMT)/radiation (RT), palliative CMT/RT and supportive care.

\section{Data collection}

Chart review was done by two physicians (TP and YT), one from each hospital, independently. For Japanese patients, they were randomly divided into 5 groups according to random numbers generated by Excel 2013 (Microsoft Co. Redmond, WA, USA) and then one of those groups was chosen. This process aimed to create 1:1 comparison. Baseline characteristics, clinical manifestations, H. pylori infection, tumor characteristics, UICC staging, treatments (resection-based vs. palliative chemotherapy/radiation vs. supportive care) and mortality rate were compared between Thai and Japanese gastric cancer patients. Moreover, factors associated with mortality from gastric cancer were assessed in patients of both countries.

\section{Statistical analysis}

Baseline descriptive data were analyzed and reported as means and standard deviations for continuous variables, and percentage and frequency for categorical variables. For univariate and multivariate analysis, continuous variables were compared using the Student's t test, and categorical variables with the chi-square (x2) test. Odd ratio (OR) was determined by using a binary logistic regression model and chi-square. Survival analysis was calculated by Kaplan Meier model and log rank testing. SPSS version 23.0 (SPSS (Thailand) Co., Ltd., Bangkok, Thailand) for Windows systems was used with differences considered significant at the 0.05 level.

\section{Results}

Participants

A total of 403 Thai and 2,318 Japanese patients were retrieved from the electronic hospital registry. The flow diagram of patient recruitment was shown in Figure 1. Finally, 332 Thais and 414 Japanese patients

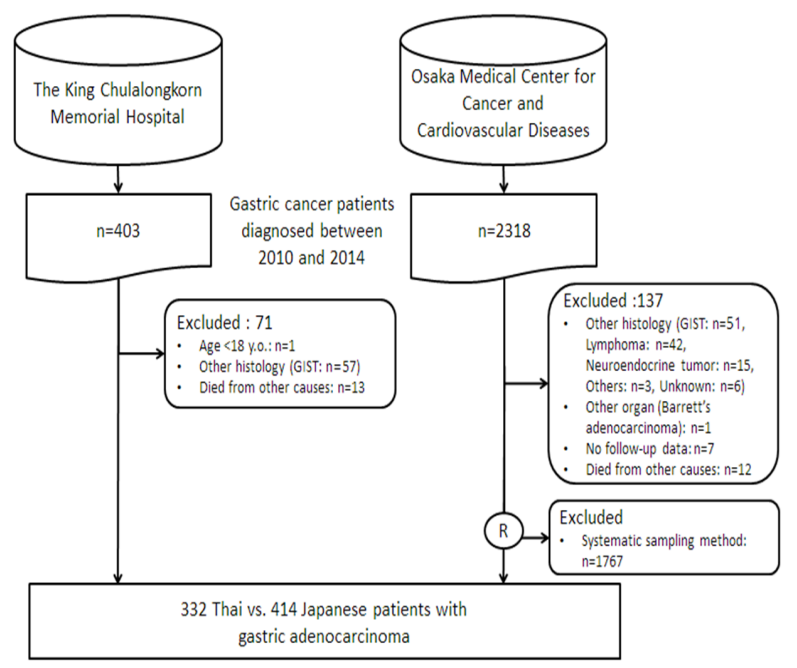

Figure1. Flow Diagram of Patient Recruitment 
Table 1. Baseline Characteristic of Patients with Gastric Adenocarcinoma

\begin{tabular}{|c|c|c|c|}
\hline & Thai patients $(\mathrm{N}=332)$ & Japanese patients $(\mathrm{N}=414)$ & P-value \\
\hline Gender, female (\%) & $181(55)$ & $102(25)$ & $<0.001$ \\
\hline Age (years \pm SD) & $59 \pm 13$ & $68 \pm 10$ & $<0.001$ \\
\hline Current smoking (\%) & $17(5.1)$ & $96(23)$ & $<0.001$ \\
\hline Current alcohol drinking (\%) & $9(2.7)$ & $143(35)$ & $<0.001$ \\
\hline Alarm symptom (\%) & $321(97)$ & $140(34)$ & $<0.001$ \\
\hline H. pylori status & & & $<0.001$ \\
\hline Positive & $146(44)$ & $75(18.1)$ & \\
\hline Negative & $39(11.7)$ & $134(32.4)$ & \\
\hline Untested & $147(44.3)$ & $205(49.5)$ & \\
\hline Extension $>2$ areas & $180(54)$ & $80(19)$ & $<0.001$ \\
\hline Clinical UICC stage (\%) & & & $<0.001$ \\
\hline Stage I & $28(8.4)$ & $340(82)$ & \\
\hline Stage II & $47(14)$ & $17(4.1)$ & \\
\hline Stage III & $76(23)$ & $14(3.4)$ & \\
\hline Stage IV & $181(55)$ & $43(10)$ & \\
\hline Pathology (\%) & & & $<0.001$ \\
\hline Well differentiated & $31(9.3)$ & $216(52)$ & \\
\hline Moderately differentiated & $44(13)$ & $98(24)$ & \\
\hline Poorly differentiated & $100(30)$ & $6(1.4)$ & \\
\hline Signet ring cell & $11(3.3)$ & $27(6.5)$ & \\
\hline Poorly differentiated + Signet ring cell & $138(42)$ & $64(16)$ & \\
\hline Unknown subtype & $8(2.4)$ & $3(0.7)$ & \\
\hline Treatment $(\%)$ & & & $<0.001$ \\
\hline ER alone & 0 & $241(58)$ & \\
\hline Surgery alone & $65(20)$ & $85(21)$ & \\
\hline ER followed by surgery & 0 & $14(3.4)$ & \\
\hline Surgery plus CMT and/or RT & $147(44)$ & $30(7.3)$ & \\
\hline CMT and/or RT & $73(22)$ & $27(6.5)$ & \\
\hline Supportive care & $47(14)$ & $17(4.1)$ & \\
\hline Follow-up time (months) & $18 \pm 18$ & $28 \pm 19$ & $<0.001$ \\
\hline Mortality rate $(\%)$ & $250(75)$ & $29(7)$ & $<0.001$ \\
\hline
\end{tabular}

were enrolled for analysis.

\section{Descriptive data}

The majority of Thai patients were female which was significantly higher than Japanese group (55\% vs. $25 \%$, $\mathrm{p}<0.001)$. Thai patients were significantly younger than Japanese patients $(59 \pm 13$ vs. $68 \pm 10$ years old, $p<0.001)$. The rate of current smoking and alcohol drinking were lower in Thai patients $(5.1 \%$ vs. $23 \%, \mathrm{p}<0.001$ and $2.7 \%$ vs. $35 \%, p<0.001$, respectively). Almost all of the Thai patients $(97 \%)$ had alarm symptoms compared with $34 \%$ of Japanese patients $(\mathrm{p}<0.001)$. H. pylori positive rate was $44 \%$ in Thais and $18.1 \%$ in Japanese $(p<0.001)$ but nearly half of the patients in both countries were neither tested nor recorded H. pylori status. Fifty-four percent of Thai patients had lesion extension more than two areas whereas only $20 \%$ were found amongst Japanese patients. Fifty-five percent of Thais presented with UICC stage IV but only $10 \%$ in Japanese patients.
Three-fourth of the Thai gastric cancer patients had poorly-differentiated adenocarcinoma and/or signet ring cell. In contrast, $75 \%$ of Japanese patients had well or moderately-differentiated adenocarcinoma. Endoscopic resection monotherapy was the main treatment in Japanese group but none of the Thais underwent this treatment before 2014 (58\%vs.0\%; p $<0.001)$. The mortality rate at the end of July 2016 was significantly higher in Thai patients $(75.3 \%$ vs. $7 \%, \mathrm{P}<0.001)$ with the mean follow-up time of $18.4 \pm 18.9$ in Thai and $28.3 \pm 19.6$ months in Japanese patients, respectively) Table 1.

\section{Outcome data and main results}

In univariate analysis, all selected parameters were significant factors of high mortality of gastric adenocarcinoma in Thailand Table 2. After adjusting all important factors by multivariate analysis, Thai ethnicity, clinical UICC stage II-IV and un-resected treatment were significant prognostic factors of high mortality rate. 

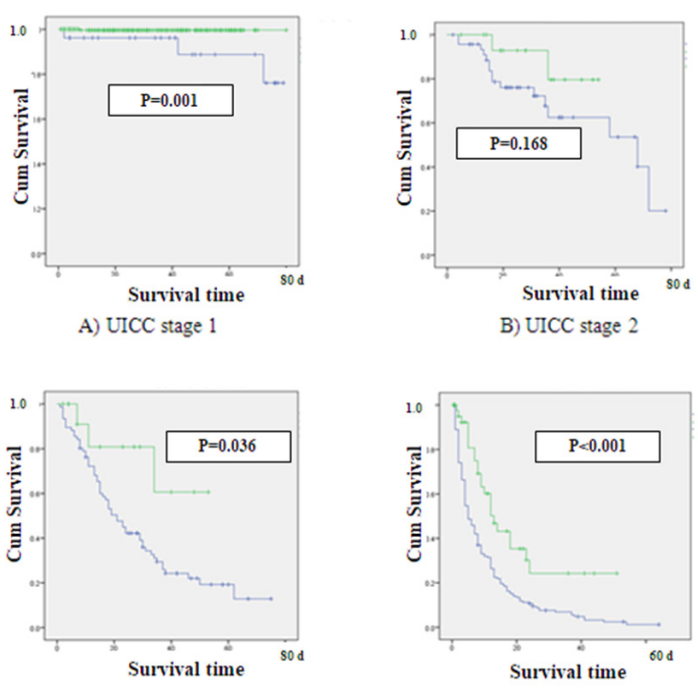

C) UICC stage 3

D) UICC stage 4

Figure 2. Survival Graph of UICC 4 Stages between Thai and Japanese Patients (Green Line: Thai Patients, Blue Line: Japanese Patients)

Clinical UICC stage IV was the strongest prognostic factor with OR 95.2 (95\%CI 26.2-346.5). Pathology provided marginal significance. Patients underwent resection-based treatment were associated with good prognosis Table 2 . Number of patients given resection-based treatment in UICC stage I-II and palliative surgery/CMT/RT in UICC stage IV were not different between Thailand and Japan Table 3.

\section{Other analyses}

The survival analysis by Keplan Meier model showed the significant better survival rate of patients with gastric adenocarcinoma stage I, III and IV in Japan than those in Thailand Figure 2.

\section{Discussion}

This is an international epidemiological-based study by using direct comparison of actual clinical data of two high-volumes gastric cancer hospitals in Thailand and Japan. This study demonstrated the significant difference in baseline characteristics of patients with gastric adenocarcinoma in both countries. Thai patients were female predominant, younger, less smoking/alcohol drinking, presented with alarm symptoms and more advanced stage of disease when compared with those in Japan. The proportion of patients who received treatment was comparable $(85 \%-100 \%$ in UICC stage I-II and 75\%-80\% in UICC stage IV); however, gastric adenocarcinoma in Thailand caused significantly higher mortality rate. After adjusting for age, gender, presence of alarm symptoms, extension of lesion and pathology of gastric adenocarcinoma; country, UICC stage and un-resected treatment were independent prognostic factors of poor survival outcome. To our knowledge, this is the first study that compared the actual clinical data between different countries.
Thai ethnicity was a strong prognostic factor for high mortality rate of gastric adenocarcinoma in this study $(\mathrm{OR}=20.1 ; \mathrm{p}<0.001$ in multivariate analysis). The difference in ethnicity has been documented as a predictor of gastric cancer in the country composing of various populations such as the United State [14-18]. They reported that the Hispanic patients had more prevalence of gastric adenocarcinoma [14, 16, 17] and higher mortality rate [17] when compared with the non-Hispanic white population. Disparity of socio-economic, healthcare assessment [19] and/or host susceptibility of H. pylori infection [20] were claimed as the etiologies of those differences in gastric adenocarcinoma between two ethnicities in the United State. In the present study, the authors did not assess the economic and healthcare status between Thailand and Japan. However, we demonstrated the same treatment rate in the two hospitals Table 3, which implies the comparable standard of healthcare system in both countries.

Nevertheless, survival was significantly higher in Japanese patients with UICC stage I, III and IV gastric adenocarcinoma. Hence, the management of gastric cancer patients in each institute was explored. The author found that two from three Thai patients with clinical UICC stage I who died from gastric adenocarcinoma were denied surgery due to elderly age at 83 years whereas most of the elderly Japanese patients underwent endoscopic resection. During study period, unfortunately, endoscopic resection had not been recognized in Thailand. Currently, there are sufficient evidences to conclude that endoscopic resection in experience institute provided similar long-term survival with fewer complication and hospital stay, compared with surgery [21, 22]. Moreover, now expert endoscopists in Thailand have experience in endoscopic resection including endoscopic submucosal resection (ESD). Consequently, we currently consider performing endoscopic resection for patients with UICC stage I gastric adenocarcinoma who could not tolerate surgery for better outcome of disease.

In late stage of UICC, even palliative chemotherapy for gastric adenocarcinoma in Thailand and Japan during 2010-2014 was similar including fluorouracil (5-FU) plus platinum-based (oxaliplatin or cisplatin) [23, 24], most of the Thai patients $(80 \%)$ with stage IV UICC staging decided to terminate their chemotherapy due to unbearable adverse events (unpublished data). In contrast, median duration of the first line treatment of Japanese patients in this study was 4.5 months with good compliance and $54 \%$ of these could proceed to the second line CMT. Thus, the management of chemotherapy toxicity should be considered in Thailand to encourage patients to complete the course of treatment for subsequent better survival rate in this particular circumstance. To clarify the impact of difference in the management of gastric cancer patients in each stage, examining in detail of diagnosis and treatment should be further evaluated. Moreover, because we suspected the difference in management of gastric cancer contributed to high mortality of patients in Thailand, centralization of patients to tertiary institution may be important to improve the quality of management 
Table 2. Univariate and Multivariate Analysis by Binary LogisticRegression Analysis of Possible Factors Associated Gigh Mortality Rate of Gastric Cancer in Thailand Compared with that of in Japan

\begin{tabular}{|c|c|c|c|c|}
\hline \multirow[t]{2}{*}{ Variables } & \multirow{2}{*}{$\begin{array}{c}\text { Univariate analysis } \\
\text { OR }(95 \% \mathrm{CI})\end{array}$} & \multicolumn{3}{|c|}{ Multivariate analysis } \\
\hline & & p-value & OR $(95 \% \mathrm{CI})$ & p-value \\
\hline Age at diagnosis & $0.95(0.94-0.96)$ & $<0.001$ & $1.00(0.98-1.02)$ & 0.69 \\
\hline Female & $2.84(2.09-3.88)$ & $<0.001$ & $0.76(0.42-1.39)$ & 0.38 \\
\hline \multicolumn{5}{|l|}{ Country } \\
\hline Japan & 1 & & 1 & \\
\hline Thai & $40.5(25.7-63.6)$ & $<0.001$ & $20.1(8.80-45.9)$ & $<0.001$ \\
\hline Current smoking & $0.25(0.15-0.43)$ & $<0.001$ & - & - \\
\hline Current alcohol drinking & $9.52(5.17-17.6)$ & $<0.001$ & - & - \\
\hline Alarm symptom & $38.5(20.0-74.4)$ & $<0.001$ & $1.57(0.46-5.40)$ & 0.47 \\
\hline \multicolumn{5}{|l|}{ H. pylori status } \\
\hline Positive & 1 & & - & - \\
\hline Negative & $0.16(0.98-0.27)$ & $<0.001$ & - & - \\
\hline Unknown & $0.71(0.50-0.99)$ & 0.045 & - & - \\
\hline \multicolumn{5}{|l|}{ Lesion extension } \\
\hline$\leq 2$ areas & 1 & & 1 & \\
\hline$>2$ areas & $6.17(4.43-8.52)$ & $<0.001$ & $0.97(0.52-1.77)$ & 0.92 \\
\hline \multicolumn{5}{|l|}{ Clinical UICC stage } \\
\hline Stage I & 1 & & 1 & \\
\hline Stage II & $35.6(11.6-110)$ & $<0.001$ & $4.85(1.39-17.0)$ & 0.01 \\
\hline Stage III & $182(61.9-535)$ & $<0.001$ & $23.8(7.01-80.6)$ & $<0.001$ \\
\hline Stage IV & $664(229-1920)$ & $<0.001$ & $95.2(26.2-347)$ & $<0.001$ \\
\hline \multicolumn{5}{|l|}{ Pathology } \\
\hline Well differentiated & 1 & & 1 & \\
\hline Non-well differentiated & $20.6(11.4-36.9)$ & $<0.001$ & $2.68(0.98-7.33)$ & 0.055 \\
\hline \multicolumn{5}{|l|}{ Treatments } \\
\hline Resection based & 1 & & 1 & \\
\hline $\mathrm{CMT} / \mathrm{RT}$ alone & $18.9(10.4-34.2)$ & $<0.001$ & $3.26(1.21-8.77)$ & 0.02 \\
\hline Supportive care & $11.0(5.89-20.4)$ & $<0.001$ & $2.35(0.78-7.03)$ & 0.13 \\
\hline
\end{tabular}

in a low-incidence country.

Another reason for the association of country and mortality rate is possibly from the difference of genetic mutation and tumor biology of gastric adenocarcinoma between two ethnic groups $[15,17,18]$. Difference in proportion of gender and histological type between two countries in the present study might reflect this possibility. A recent report in epidemiologic study revealed a higher mortality rate of gastric cancer amongst Asian American (Koreans, Japanese and Chinese) than non-Hispanic white population (10-20\% vs. less than $2 \%$ ) [18]. Thus, further basic-science research in gene/mutation of gastric adenocarcinoma should be initiated to prove this hypothesis.

Indeed, even adjusted for other variables including country, clinical UICC stage IV was the strongest significant factor of high mortality rate of gastric cancer in this study. It emphasized the fact that early diagnosis is fundamentally important for better survival on gastric adenocarcinoma [25]. This study showed that $97 \%$ of Thai patients presented with gastric cancer usually had alarm symptoms and were found to be in advanced stages (Stage III+Stage IV: 78\%). In contrast, 66\% of Japanese patients with gastric adenocarcinoma did not have any alarm symptoms and UICC stage I was accounted for $82 \%$. Implementation of population-based screening contributes to early detection of gastric cancer in Japan. However, in a leading cancer center, only $7.6 \%$ of their patients were found to be early gastric cancer, while the majority $(>90 \%)$ were detected outside of population-based screening program (from outpatient clinic at $64 \%$ and individual health-check at 28\%) [26]. Moreover, because of high incidence of gastric cancer in Japan, most people are aware of the importance of early gastric cancer diagnosis. They often have siblings or relatives suffering from gastric cancer, and attended outpatient clinics to receive endoscopy with very mild or even no symptom. Availability of social health insurance system and large number of endoscopists in Japan allow Japanese patients to easy access of endoscopy. Therefore, efficient strategies to identify high-risk patients for screening and surveillance endoscopy should be established in country with low-prevalence of gastric cancer. 
Table 3. Treatment in Patients with Gastric Adenocarcinoma in Each UICC Staging in both Countries

\begin{tabular}{lccc}
\hline UICC staging & Thai patients (N=332) & Japanese patients (N=414) & p-value \\
\hline Stage I (N=368) with endoscopic or surgical resection plus CMT/RT & $92.9 \%$ & $97.9 \%$ & 0.144 \\
Stage II (N=64) with surgical resection plus CMT/RT & $95.7 \%$ & $100 \%$ & 1.000 \\
Stage III (N=90) with surgical resection with/without CMT/RT & $92.1 \%$ & $71.4 \%$ & 0.045 \\
Stage IV (N=224) with palliative surgery or palliative CMT/RT & $75.7 \%$ & $79.1 \%$ & 0.695 \\
\hline
\end{tabular}

Although we found difference in proportion of histological type between Thai and Japan, this could be attributed to the different criteria in the "main histological type" diagnosis. Japanese classification regards the prominent histological type as the "main histological type" while Western criteria used the highest grade [11, 12]. In addition, histology provided a marginally significant association with mortality in this study, showing better survival rate in patient with well differentiated adenocarcinoma. In Japan, high grade dysplasia is usually involved as well-differentiated early gastric cancer [12]. Disparity in pathology classification in early stage of gastric adenocarcinoma may affect the potential different outcome.

H. pylori is a well-established carcinogen for gastric cancer [27]. The authors tried to evaluate the difference in H. pylori infection and eradication rate. Unfortunately, half of the patients with gastric adenocarcinoma in both countries were not tested for $\mathrm{H}$. pylori status; hence, the data of $\mathrm{H}$. pylori infection was not assessed in multivariate analysis and discussed as a significant associated factor. However, previous information established high prevalence of $\mathrm{H}$. pylori infection in both Thailand and Japan at approximately $50 \%-70 \%[28,29]$. Therefore, the prevalence of $\mathrm{H}$. pylori infection should not be an influential factor of the difference in mortality rate in these two countries. Virulence or host susceptibility of $\mathrm{H}$. pylori infection should be further evaluated to address the associated poor prognosis of gastric adenocarcinoma in Thailand.

This study had some certain limitations. First, this is a retrospective study. Some particular information such as family history of gastric cancer, H. pylori infection and tumor size were still lacking due to incomplete data record. Despite this predictable flaw in retrospective study, this type of research still has its value and needed to define basic epidemiological differences. Second, the authors could not classify "endoscopic resection (ER)" as an independent category because none of Thai patients received ER. However, we grouped ER, surgical resection and surgical resection plus CMT/RT as "resection-based" treatment which represent the curative purpose. Finally, the authors did not match neither age nor gender before analysis. However, this study was originally designed as an epidemiological-based study to evaluate actual data between high- and low-prevalence countries of gastric cancer. The case-control matched method is considered for the future study.

In conclusion, the clinicopathological data of patients with gastric adenocarcinoma in Thailand and Japan were totally different. Country, clinical UICC staging II-IV compared to I and un-resected treatment method were the major prognostic factors for high mortality rate. Only direct adaptation of Japanese gastric cancer screening to Thailand may not be sufficient to improve outcome of Thai gastric cancer patients. Further evaluation in patient management including diagnosis, treatment and tumor biology are essential to find the individual strategy to improve survival of gastric adenocarcinoma in a low-prevalence area such as Thailand in the future.

\section{Acknowledgements}

Authors send gratitude to Drs. Isao Miyashiro, Naotoshi Sugimoto, Minoru Kato, Kenta Hamada, Yasushi Yamasaki, Noriko Matsuura, Takashi Kanesaka for significant assistants for study preparation and data collection and Miss Chonlada Phathong for confirming data analysis.

\section{Funding Statement}

This research was funded by the Grant for International Research Integration: Chula Research Scholar, Ratchadaphiseksomphot Endowment Fund.

\section{References}

1. Allemani C, Weir HK, Carreira H, Harewood R, Spika D, Wang XS, et al. Global surveillance of cancer survival 1995-2009: analysis of individual data for 25,676,887 patients from 279 population-based registries in 67 countries (CONCORD-2). Lancet (London, England). 2015;385(9972):977-1010.

2. Torre LA, Bray F, Siegel RL, Ferlay J, Lortet-Tieulent J, Jemal A. Global cancer statistics, 2012. CA: a cancer journal for clinicians. 2015;65(2):87-108.

3. Nanthanangkul S, Suwanrungruang K, Wiangnon S, Promthet S. Survival of Stomach Cancer Cases in Khon Kaen, Thailand 2000-2012. Asian Pacific journal of cancer prevention : APJCP. 2016;17(4):2125-9.

4. Isobe Y, Nashimoto A, Akazawa K, Oda I, Hayashi K, Miyashiro I, et al. Gastric cancer treatment in Japan: 2008 annual report of the JGCA nationwide registry. Gastric cancer : official journal of the International Gastric Cancer Association and the Japanese Gastric Cancer Association. 2011;14(4):301-16.

5. Ferlay J, Soerjomataram I, Dikshit R, Eser S, Mathers C, Rebelo M, et al. Cancer incidence and mortality worldwide: sources, methods and major patterns in GLOBOCAN 2012. International journal of cancer. 2015;136(5):E359-86.

6. Tan YK, Fielding JW. Early diagnosis of early gastric cancer. European journal of gastroenterology \& hepatology. 2006;18(8):821-9.

7. Asaka M. A new approach for elimination of gastric cancer deaths in Japan. International journal of cancer. 2013;132(6):1272-6.

8. Hamashima C, Ogoshi K, Narisawa R, Kishi T, Kato $\mathrm{T}$, Fujita K, et al. Impact of endoscopic screening on 
mortality reduction from gastric cancer. World journal of gastroenterology. 2015;21(8):2460-6.

9. Naylor GM, Gotoda T, Dixon M, Shimoda T, Gatta L, Owen $\mathrm{R}$, et al. Why does Japan have a high incidence of gastric cancer? Comparison of gastritis between UK and Japanese patients. Gut. 2006;55(11):1545-52.

10. Institue. NC. Stomach (Gastric) Cancer Prevention (PDQ(R)): Health Professional Version. Bethesda. http:// www.ncbi.nlm.nih.gov/pubmed/26389263.(2002): [

11. Lauwers GY CF, Graham DY Gastric carcinoma. In 'Classification of Tumours of the Digestive System', Eds Bowman FT, Carneiro F and Hruban RH. IARC, Lyon (2010). .

12. Japanese classification of gastric carcinoma: 3rd English edition. Gastric cancer : official journal of the International Gastric Cancer Association and the Japanese Gastric Cancer Association. 2011;14(2):101-12.

13. Sobin LH GM, Wittekind $\mathrm{CH}$ e. In 'International Union Against Cancer (UICC) TNM Classification of Malignant Tumors. 7ed.', Eds Sobin LH, Gospodarowicz MK and CH W. (2009).

14. Yao JC, Tseng JF, Worah S, Hess KR, Mansfield PF, Crane $\mathrm{CH}$, et al. Clinicopathologic behavior of gastric adenocarcinoma in Hispanic patients: analysis of a single institution's experience over 15 years. J Clin Oncol. 2005;23(13):3094-103.

15. Trinh QD, Nguyen PL, Leow JJ, Dalela D, Chao GF, Mahal BA, et al. Cancer-specific mortality of Asian Americans diagnosed with cancer: a nationwide population-based assessment. Journal of the National Cancer Institute. 2015;107(6):djv054.

16. Dong E, Duan L, Wu BU. Racial and Ethnic Minorities at Increased Risk for Gastric Cancer in a Regional US Population Study. Clinical gastroenterology and hepatology : the official clinical practice journal of the American Gastroenterological Association. 2017;15(4):511-7.

17. Duma N, Sanchez LJ, Castro YS, Jennis AA, McCain DA, Gutierrez ME, et al. Gastric adenocarcinoma: clinicopathologic differences among Hispanics and nonHispanic whites. A single Institution's experience over 14 years. Annals of gastroenterology. 2016;29(3):325-31.

18. Thompson CA, Gomez SL, Hastings KG, Kapphahn K, Yu P, Shariff-Marco S, et al. The Burden of Cancer in Asian Americans: A Report of National Mortality Trends by Asian Ethnicity. Cancer epidemiology, biomarkers \& prevention : a publication of the American Association for Cancer Research, cosponsored by the American Society of Preventive Oncology. 2016;25(10):1371-82.

19. Derose KP, Escarce JJ, Lurie N. Immigrants and health care: sources of vulnerability. Health affairs (Project Hope). 2007;26(5):1258-68.

20. Malaty HM, Engstrand L, Pedersen NL, Graham DY. Helicobacter pylori infection: genetic and environmental influences. A study of twins. Annals of internal medicine. 1994;120(12):982-6.

21. Cho JH, Cha SW, Kim HG, Lee TH, Cho JY, Ko WJ, et al. Long-term outcomes of endoscopic submucosal dissection for early gastric cancer: a comparison study to surgery using propensity score-matched analysis. Surgical endoscopy. 2016;30(9):3762-73.

22. Ryu SJ KB, Kim BG, et al Endoscopic submucosal dissection versus surgical resection for early gastric cancer: a retrospective multicenter study on immediate and long-term outcome over 5 years. . Surg Endosc, . (2016). ; 30,:5283-9.

23. Waters JS, Norman A, Cunningham D, Scarffe JH, Webb A, Harper P, et al. Long-term survival after epirubicin, cisplatin and fluorouracil for gastric cancer: results of a randomized trial. British journal of cancer. 1999;80(1-2):269-72.

24. Koizumi W, Narahara H, Hara T, Takagane A, Akiya T, Takagi M, et al. S-1 plus cisplatin versus S-1 alone for firstline treatment of advanced gastric cancer (SPIRITS trial): a phase III trial. The Lancet Oncology. 2008;9(3):215-21.

25. Graziosi L, Marino E, Cavazzoni E, Donini A. Prognostic value of the seventh AJCC/UICC TNM classification of noncardia gastric cancer. World journal of surgical oncology. 2013;11:103.

26. Suzuki H, Gotoda T, Sasako M, Saito D. Detection of early gastric cancer: misunderstanding the role of mass screening. Gastric cancer : official journal of the International Gastric Cancer Association and the Japanese Gastric Cancer Association. 2006;9(4):315-9.

27. Nardone G, Staibano S, Rocco A, Mezza E, D'Armiento F $\mathrm{P}$, Insabato L, et al. Effect of Helicobacter pylori infection and its eradication on cell proliferation, DNA status, and oncogene expression in patients with chronic gastritis. Gut. 1999;44(6):789-99.

28. Sahara S, Sugimoto M, Vilaichone RK, Mahachai V, Miyajima H, Furuta T, et al. Role of Helicobacter pylori cagA EPIYA motif and vacA genotypes for the development of gastrointestinal diseases in Southeast Asian countries: a meta-analysis. BMC infectious diseases. 2012;12:223.

29. Uchida T, Miftahussurur M, Pittayanon R, Vilaichone RK, Wisedopas N, Ratanachu-Ek T, et al. Helicobacter pylori Infection in Thailand: A Nationwide Study of the CagA Phenotype. PloS one. 2015;10(9):e0136775.

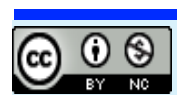

This work is licensed under a Creative Commons AttributionNon Commercial 4.0 International License. 